\title{
Migraciones internas hacia la Región Metropolitana de Santiago de Chile: una comparación con planteamientos teóricos.*
}

\author{
Paulina Schiappacasse Cambiaso \\ Departamento de Geografía, Universidad de Chile \\ pschiapp@uchile.cl \\ Miguel Contreras Alonso \\ Departamento de Geografía, Universidad de Chile \\ mcalonso@ctcreuna.cl \\ Carlos Fuensalida Claro \\ Departamento de Urbanismo, Universidad de Chile
}

Recibido 30 diciembre 2001; versión corregida aceptada, 24 octubre 2002.

\begin{abstract}
RESUMEN
Se analiza la orientación de los movimientos migratorios en Chile, los cuales están implícitos en el debate actual sobre las causas del crecimiento demográfico del Gran Santiago. En éste se resalta, por un lado, la atracción que puede continuar presentando la metrópolis y por otro, una tipología de inmigrante poco selectiva. Se determina y evalúa, con una resolución espacial comunal, el impacto de los movimientos migratorios regionales en el crecimiento de la ciudad, identificando el perfil que caracteriza al inmigrante hacia fines de la década de los 80 . Los resultados muestran que el aporte poblacional desde regiones, en términos cuantitativos, resulta ser menos significativo que los movimientos intraurbanos registrados en el mismo período. La interacción espacial, se manifiesta en una fuerte asociación con las regiones centrales del país y un patrón de destino metropolitano difuso, centro-periferia. Se trata de una migración selectiva, que se manifiesta con particular fuerza en las comunas de mayor status socioeconómico. Corresponde a un modelo de comportamiento ya encontrado para Latinoamérica y sistematizado en un paradigma de la urbanización. La Región Metropolitana de Santiago ha perdido peso en su rol histórico, polarizador de movimientos; sin embargo, prosigue la tendencia a la aglomeración, en el marco de una nueva jerarquía funcional: el dinamismo experimentado por las ciudades intermedias.
\end{abstract}

Palabras clave: migraciones interregionales, concentración urbana, planificación urbano-regional.

\section{Internal Migrations towards the Metropolitan Region of Santiago, Chile: A confrontation with theoretical approaches*}

\begin{abstract}
The orientations of migrations movement patterns in Chile are analyzed, with special reference to the causes of the demographic growth of the Greater Santiago Area. On the one hand, these are related to the degree of attraction that the area still has within the country, and on the other, with a predominant nonselective emigrant typology. It was determinate, in a communal level of resolution, the impact of the regional migrations movements in the city growth, by means of the identification of the 1980's immigrant profile. The results indicate that the influx from other regions is definitely less significant than the intra - regional movements in the same period. Spatial interaction reveals a strong relationship with the central regions of the country, as well as a diffuse destiny pattern central - peripheral within the metropolis. Associated attributes indicate a very selective movement trend that stands out in the wealthier communes. The results identify a behavioral pattern already analyzed in an urbanization paradigm for Latin America. The Metropolitan Region of Santiago has lost the historical polarizing role of movements. Nevertheless, the tendency to the agglomeration continues, in a new functional hierarchic framework: the dynamism of the mid size cities.
\end{abstract}

Key words: inter-regional migration movements, urban agglomeration, urban-regional planning.

* Proyecto financiado por la Facultad de Arquitectura y Urbanismo de la Universidad de Chile. 


\section{INTRODUCCION}

Los flujos migratorios forman parte inseparable de la historia de América Latina. La Región es el producto de oleadas de inmigrantes que histórica y espacialmente se han manifestado en tres tipos de flujos: la inmigración de ultramar, con particular fuerza entre mediados del siglo XIX e inicios del siglo XX; la migración interna, entre diversos países, una constante en la historia social de la Región y la emigración hacia el exterior. Independientemente de la tipología de movilidad espacial que se ha materializado en Latinoamérica, los flujos han tenido un mismo destino, las áreas urbanas, constatándose un extraordinario crecimiento de la población residente en las metrópolis y ciudades intermedias de la región.

En efecto, el espacio geográfico latinoamericano es - en la actualidad - la Región más intensamente urbanizada del mundo en vías de desarrollo y, según todas las previsiones, continuará siéndolo, pasando de una tasa de población urbana de $67 \%$ en 1986 a una de $75 \%$ en el año 2000 . Se puede decir que tres de cada cuatro personas viven en asentamientos urbanos (RODRIGUEZ \& VILLA 1998). Si bien la rápida urbanización de la región es un proceso reconocido, no ha de olvidarse la notable diversidad interna que la caracteriza, debido a que los procesos evolutivos sociales, políticos y económicos de cada país han sido disímiles. En efecto, países como El Salvador, Honduras, Costa Rica y Guatemala, presentan a la fecha montos de urbanización inferiores al $50 \%$, mientras que - en el extremo opuesto - destacan Chile, Argentina, Uruguay y Venezuela con porcentajes de población urbana superiores al $80 \%$ (INSTITUTO LATINOAMERICANO DE PLANIFICACIÓN ECONÓMICA Y SOCIAL, ПLPES 2000).

Esta elevada urbanización, que de manera generalizada se ha venido registrando en América Latina, y con mayor intensidad en los países del Cono Sur, no se atribuye a ninguna de las tres tipologías presentadas con an- terioridad, sino más bien a los movimientos migratorios internos. En este sentido, hay consistencia con los resultados compilados por PUYOL (1993), relativos a que cuando una alta proporción de la población vive en zonas urbanas, los movimientos interurbanos tienden a reemplazar el éxodo rural como causal principal de movilidad interna. En definitiva se observa una modificación de los patrones migratorios dominantes en la Región, lo que se ha materializado en una pérdida de importancia de los flujos rural-urbanos, acentuándose el peso de la movilidad intra e interurbana.

En el caso de Chile, la distribución espacial de la población se caracteriza por una progresiva integración de los diferentes territorios en un sistema de economía urbana dominante, con $86 \%$ de población urbana, según el último Censo de Población 2002 (INSTITUTO NACIONAL DE ESTADÍSTICAS, INE 2002), lo que ha tenido como consecuencia el desencadenamiento de un proceso creciente de movilidad interior, el que ha experimentado transformaciones significativas en las últimas décadas. El Gran Santiago (Fig. 1), tras una primera etapa de polarización de movimientos regionales, derivada de la implantación del modelo económico de sustitución de importaciones, ha desarrollado una modalidad de crecimiento extensiva en la última década, que responde a un predominio de los movimientos internos, con fuertes pérdidas poblacionales en el anillo central y crecimiento en localizaciones periféricas (SCHIAPPACASSE 1998).

Asociado a lo expuesto, la ciudad de Santiago ha experimentado modificaciones, reflejadas en bajas tasas de natalidad y mortalidad, aumento en la expectativa de vida y una disminución en el número de hijos. Cambios notables reflejan las tasas netas de natalidad y mortalidad en el período 1950-2002, que indican que el paîs se encuentra en un proceso de plena transición demográfica; en el primer caso el descenso ha sido de 34.8 a 21.7 por mil, mientras que, la mortalidad ha disminuido de 15.7 a 5.5 por mil. La esperanza 
de vida al nacer se ha incrementado en aproximadamente 20 años desde 1950; en la actualidad ésta alcanza a poco más de 68 años en el caso de los hombres y 75 en las mujeres. La fecundidad se ha reducido desde aproximadamente 5 hijos por mujer durante el período 1950-55 a 2.7 hijos en el quinquenio 1990-95 (CENTRO LATINOAMERICANO DE DEMOGRAFÍA, CELADE 1991). Este patrón coincide con las observaciones de ZELINSKI (en COURGEAU 1986), sobre la existencia de una fuerte asociación entre el patrón de movilidad interna nacional y el estadio de transición demográfica.

En definitiva, la ciudad presenta una modalidad de crecimiento en la última década que se puede tipificar como extensiva, horizontal, francamente dinámica, que responde más a un predominio de los movimientos internos y a cambios en el ciclo de vida de la población (ORTIZ \& SCHIAPPACASSE 1997), que al peso de las corrientes inmigratorias regionales. Estas modificaciones en la orientación, destino y características de los flujos internos son el objetivo que orienta esta investigación.

\section{MATERIALES Y METODOS}

Existen diferencias evidentes entre las definiciones asociadas al concepto de migración, las que no solo abarcan el campo de lo conceptual, sino también el operacional. Por lo tanto, las cuestiones de definición de migración y las fuentes de datos migratorios están fuertemente entrelazadas. En este contexto, el concepto de migración es más específico que la idea de movilidad geográfica o espacial de una población (SASSONE 1991).

En esta investigación, se considera dos elementos básicos el proceso migratorio: la población migrante y el territorio desde y hacia dónde se desplaza. Ambos están condicionados por variados aspectos tales como los factores causales (estructurales o coyunturales), las características intrínsecas del proceso (volumen y distancia) y las características propias de la población migrante (selectividad).
La información principal fue obtenida del censo de 1992 (INSTITUTO NACIONAL DE ESTADÍSTICAS, INE 1992), el cuál consideró tres preguntas para medir el fenómeno migratorio: a) lugar de nacimiento, b) lugar de residencia habitual y c) lugar de residencia 5 años antes del momento censal. Las dos primeras estuvieron dirigidas a toda la población y la tercera a la población de 5 años y más de edad. Para efectos de esta investigación, migrante es aquella persona que tiene su residencia habitual en una comuna distinta a la que tenía 5 años antes de la aplicación del censo, es decir en el año 1987.

El censo entrega los datos de migración sobre la base de dos variables: lugar de nacimiento del migrante y período de migración con respecto a unidades político - administrativas mayores (comunas). Las preguntas descritas con anterioridad encierran un número de problemas que ARÉVALO (1985), agrupa del siguiente modo: problemas que afectan la calidad de los datos derivados del diseño del formulario, de los errores de los encuestadores y de la elaboración inadecuada de los antecedentes recogidos. Lo cierto es que las fuentes censales presentan limitaciones o imperfecciones, que restringen la interpretación del fenómeno. En relación con lo anterior, queda claro que la fuente de datos a la cual se recurre no permite ahondar en los factores causales que condicionaron el proceso de cambio residencial. Por lo tanto, los factores explicativos tendrán relación con las disparidades territoriales tanto en el origen como en el destino del movimiento.

En este contexto y para el análisis de los factores de carácter económico que expliquen el comportamiento de los flujos migratorios hacia el Gran Santiago, se consideró, en particular, la evolución de tres indicadores para el período 1985-1992: producto interno bruto (PIB); población económicamente activa (PEA) y tasa de desempleo (PASSARIS 1989). El análisis de estos indicadores fue complementado con una cuarta variable: la oferta de un servicio de primer orden como lo es el número de matrículas en educación 
superior. En efecto, de acuerdo con la tipología etaria predominante, la alta proporción de población joven puede ser causante de flujos migratorios. Finalmente, se incorporan en la discusión final indicadores no económicos, como el indice de desarrollo humano, las disparidades en el ingreso y la perspectiva de género (COMISIÓN ECONÓMICA PARA AMÉRICA LATINA, CEPAL 1996), para complementar los anteriores y entender cuales son las fuerzas que operan detrás de estos movimientos.

La información levantada por INE (1992), fue analizada a un nivel de resolución espacial comunal, para lo cual se utilizó el software REDATAM+, el que permite manejar los atributos asociados - sexo, nivel educativo, condición de ocupación, lugar de nacimiento - a la población que en 1992 declaró residir fuera de la Región Metropolitana en 1987.

\section{RESULTADOS}

\section{Patrón de Origen}

Si se considera que la capital de Chile concentra el $35 \%$ de la población, no es de extrañar que durante el período 1987-1992 la Región Metropolitana haya polarizado el $30 \%$ de los movimientos interregionales (Fig. 1, Tabla 1 y Fig. 2). En efecto en el período señalado se registraron 243.991 movimientos inmigratorios, cifra levemente inferior, en términos absolutos, a la registrada en el período censal anterior. Sin embargo, resulta interesante constatar por un lado, que prácticamente se ha duplicado el número de movimientos en el país, y por otro, que en términos relativos ha descendido el rol polarizador del Area Metropolitana de un 40 a un 30\%, considerando el total de movimientos realizados.

\section{Tabla 1. Evolución de los movimientos hacia la Región Metropolitana Table 1. Evolution of The Migratory MOVEMENTS toward the Metroplitan REGIÓN}

\begin{tabular}{|c|c|c|c|}
\hline Periodo Censal & Total inmigrates & Total movimientos pais & $\%$ \\
\hline $1965-1970$ & 220.348 & 534.765 & 41.2 \\
\hline $1977-1982$ & 244.368 & 594.867 & 41.0 \\
\hline $1987-1992$ & 243.991 & 800.940 & 30.4 \\
\hline
\end{tabular}

Fuente: Censos INE: 1970, 1982, 1992.

Estas cifras indican un cambio con respecto a lo encontrado por ELIZAGA (1968), quien - al analizar los resultados de una encuesta realizada en el Gran Santiago durante1962 destaca la importancia numérica de la migración hacia Santiago. Alrededor del 50\% de la población de 15 años o más provenía de fuera del Area Metropolitana. Esta modificación en los montos de los flujos responde según CELADE (1999), al fuerte dinamismo demográfico experimentado por 15 ciudades intermedias- comunas o conglomerados de comunas con más de 100.000 habitantes urbanos en 1992, las que concentraron el $45.9 \%$ del total de movimientos, destacando además del Gran Valparaíso $(7,0 \%)$ y el Gran Concepción (6,3\%), las ciudades de Temuco $(4,0 \%)$, Antofagasta $(3,8 \%)$ y la conurbación Coquimbo - La Serena $(3,4 \%)$.

De más de 200 mil movimientos que se registraron hacia la Región Metropolitana (compuesta por 52 comunas), más del $90 \%$ se efectuó hacia alguna de las 34 comunas que componen el Gran Santiago, cifra que refleja las diferencias en términos de atracción que ejerce esta entidad con relación a su periferia, la que a la fecha aún registra población rural, bajas densidades y una tendencia a la concentración de la población en las respectivas ciudades cabeceras comunales. 


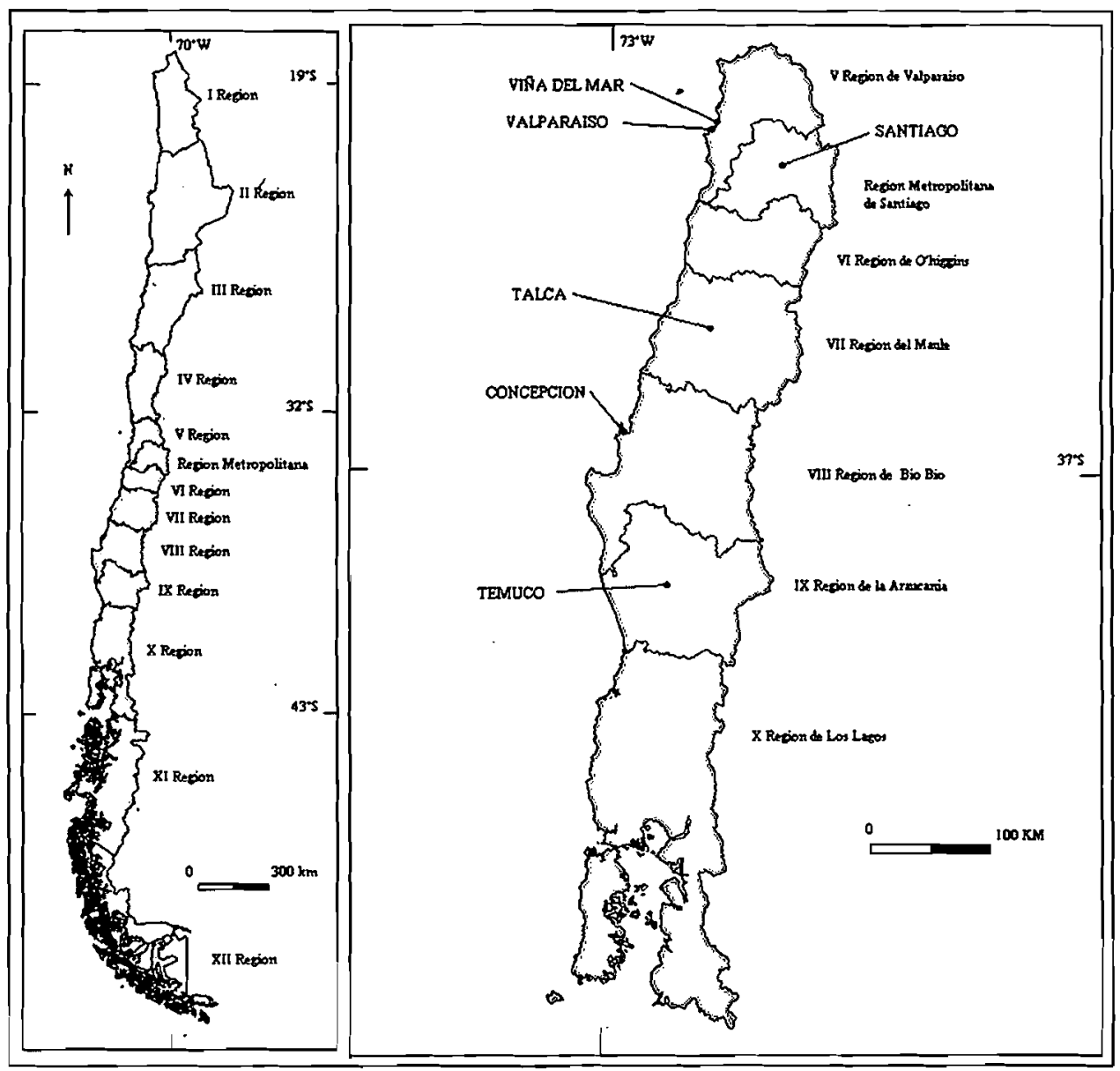

Fig. 1. Mapa de ubicación de las regiones analizadas en Chile. Gig. 1. Locational map of the analyzed regions in Chile.

En cuanto al origen de la población migrante, la Tabla 2.da cuenta de un decaimiento de la interacción espacial a medida que aumenta la distancia. Se comprueba entonces una de las leyes de RAVENSTEIN (1889), que señala que la mayor parte de los movimientos migratorios son de corta distancia.. En efecto, los mayores aportes registrados en el período 1987-1992, se producen desde las regiones del Bio-Bío, Valparaíso y del Maule, las que en su conjunto concentran casi el $50 \%$ del flujo inmigratorio total. Interesante resulta comparar cómo se ha modificado el patrón de origen desde la década de los 60 , donde la región centro sur del país (VI a $\mathrm{IX}$ regiones) concentraba más del $70 \%$ de los aportes, destacando la región de la Araucanía con el se- gundo flujo más relevante (Fig. 1). Sin embargo, desde fines de la década de los 60 , la VIII Región se mantiene como el espacio que aporta el mayor porcentaje de migrantes (Tabla 2).

Los factores pueden estar asociados con la paulatina fragmentación del polo de desarro1 lo industrial en el área de Concepción (Fig. 1) y la disminución de las actividades extractivas de carbón en la parte sur de ésta, situación que tiende a agudizarse a principios de la década de los 80. En otras palabras, se relacionan con el progresivo abandono de las industrias de los centros industriales tradicionales con gran intensidad en fuerza de trabajo, ya anticuadas. 
Tabla 2. Evolución de los flujos migratorios hacia la Región Metropolitana (\%), Fig. 1

TABle 2. Evolution Of the Migratory flows toward the Metropolitan Region (\%), Fig. 1

\begin{tabular}{|c|c|c|c|}
\hline Región & 1965.70 & $1977-82$ & 1987.92 \\
\hline I & 1.2 & 3.7 & 4.9 \\
\hline II & 2.0 & 4.1 & 4.6 \\
\hline III & 1.0 & 1.9 & 1.8 \\
\hline V & 4.1 & 3.7 & 4.1 \\
\hline V & 8.7 & 13.7 & 15.3 \\
\hline VI & 12.5 & 10.7 & 10.1 \\
\hline VII & 15.2 & 13.6 & 13.0 \\
\hline VIII & 22.4 & 22.0 & 20.3 \\
\hline IX & 20.0 & 12.3 & 11.8 \\
\hline$X$ & 12.0 & 12.3 & 10.8 \\
\hline$X I$ & 0.2 & 0.6 & 0.9 \\
\hline$X I I$ & 0.7 & 1.4 & 2.4 \\
\hline Total & 100.0 & 100.0 & 100.0 \\
\hline
\end{tabular}

Fuente: Rodriguez, 1995, INE, 1992

Si se investiga a un nivel de resolución mayor cuáles son las comunas que registran los aportes más significativos, es posible constatar, por un lado, el fuerte sesgo urbano de los flujos (sobre el $70 \%$ en las tres regiones «más expulsoras»), y por otro, las constitución de las capitales regionales en el foco principal de emigración (Tabla 3).

Tabla 3. Principales flujos migratorios según origen urbano (\%), Fig. 1

Table 3. Principal migratory flows related to urban origin (\%), Fig. 1

\begin{tabular}{|c|c|}
\hline Región /Comuna & $\%$ \\
\hline V Región & 96.8 \\
\hline Viña del Mar & 25,5 \\
\hline Valparaiso & 16.7 \\
\hline San Antonio & 8.8 \\
\hline Los Andes & 6.3 \\
\hline VII Región & 73.9 \\
\hline Talca & 22,7 \\
\hline Linares & 12,6 \\
\hline Cauquenes & 7.7 \\
\hline
\end{tabular}

\begin{tabular}{|c|c|}
\hline Región /Comuna & $\%$ \\
\hline VIII Región & $\mathbf{8 5 . 0}$ \\
\hline Concepción & 20,9 \\
\hline Los Angeles & 9.7 \\
\hline Parral & 7.2 \\
\hline Chillán & 15,5 \\
\hline Talcahuano & 5.5 \\
\hline San Carlos & 5.2 \\
\hline Coronel & 6.3 \\
\hline
\end{tabular}

Fuente: INE, 1992 


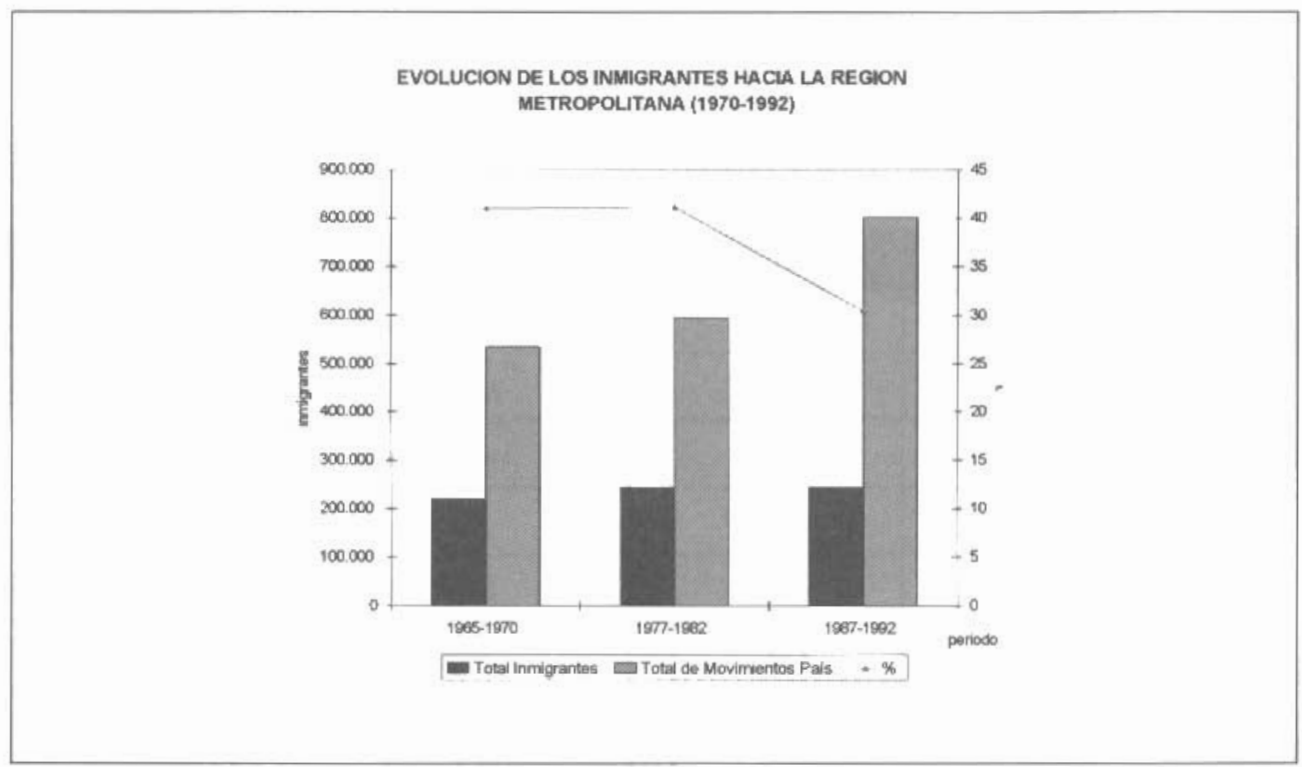

Fig. 2. Evolución de los movimientos migratorios interregionales de Chile en tres períodos. El total de movimientos ha aumentado, mientras que el número de desplazamientos hacia la Región Metropolitana de Santiago se ha mantenido constante; por ello la proporción de estos últimos ha perdido importancia relativa entre 1965 y 1992.

Fig. 2. Evolution of interregional migratory movements in Chile in three periods. The total of movements shows a constant increase, but the movements directed to Santiago Metropolitan Region has been relatively stabilized; due to that the last one are less significant in 1992 than 1965.

El patrón de destino

El patrón de destino se materializa espacialmente en una estructura centro-periferia, con estribaciones hacia el sur oriente y poniente de la ciudad, adquiriendo el Gran Santiago más peso que su periferia suburbana circundante (Figs. 3 y 4)). La comuna de Santiago polarizó la mayor cantidad de movimientos (9\%), seguida por Las Condes, La Florida, Providencia, Maipú, Ñuñoa, Puente Alto. Estación Central, Peñalolén y San Bernardo. Estas 10 entidades recibieron más de la mitad de los movimientos registrados hacia la región (Tabla 4).

De esta forma, la entidad central de la ciudad - comuna de Santiago, foco cultural, financiero y de servicios - continúa siendo el es- pacio de mayor interés para el inmigrante, tal como se constata en los trabajos de ELIZAGA (1970) y del INSTITUTO DE PATOLOGIA SOCIAL (1968).

Segregación espacial en la orientación de los flujos

Paralelamente se trató de indagar, a un nivel de resolución espacial mayor (distrito), si existía una segregación espacial en la orientación de los flujos; en otras palabras si era posible distinguir distritos, al interior del Gran Santiago, que registraran un mayor poder de atracción para los inmigrantes regionales (se excluyen las restantes 18 comunas de la Región Metropolitana, de bajo peso inmigratorio). 
Tabla 4. Destino de los flujos migratorios hacia la

Región Metropolitana (\%)

Table 4. Migratory flows destination toward the Metropolitan Region (\%)

\begin{tabular}{|c|c|}
\hline Comuna & $\%$ \\
\hline Santiago & 8,2 \\
\hline Las Condes & 7,54 \\
\hline La Florida & 6,17 \\
\hline Providencia & 4,80 \\
\hline Maipú & 4,53 \\
\hline Ñuñoa & 4,51 \\
\hline Puente Alto & 4,03 \\
\hline E. Central & 3,14 \\
\hline Peñalolén & 3,12 \\
\hline San Bernardo & 2,97 \\
\hline Vitacura & 2,62 \\
\hline Macul & 2,51 \\
\hline La Reina & 2,50 \\
\hline El Bosque & 2,43 \\
\hline La Pintana & 2,22 \\
\hline Pudahuel & 2,21 \\
\hline Cerro Navia & 2,17 \\
\hline Recoleta & 2,16 \\
\hline Conchali & 1,96 \\
\hline Lo Prado & 1,93 \\
\hline La Granja & 1,91 \\
\hline Quinta Normal & 1,86 \\
\hline Renca & 1,84 \\
\hline Lo Barnechea & 1,77 \\
\hline La Cisterna & 1,75 \\
\hline San Miguel & 1,66 \\
\hline
\end{tabular}

\begin{tabular}{|c|c|}
\hline Comuna & $\%$ \\
\hline P. Aguirre Cerda & 1,59 \\
\hline San Joaquín & 1,58 \\
\hline Independencia & 1,56 \\
\hline Cerrillos & 1,41 \\
\hline San Ramón & 1,39 \\
\hline Lo Espejo & 1,23 \\
\hline Colina & 1,09 \\
\hline Buin & 0,93 \\
\hline Melipilla & 0,92 \\
\hline Peñaflor & 0,85 \\
\hline Paine & 0,78 \\
\hline Quilicura & 0,65 \\
\hline Huechuraba & 0,63 \\
\hline Talagante & 0,55 \\
\hline Lampa & 0,44 \\
\hline Tiltil & 0,33 \\
\hline Curacavi & 0,27 \\
\hline Pirque & 0,23 \\
\hline Calera de Tango & 0,22 \\
\hline San José de Maipo & 0,19 \\
\hline El Monte & 0,19 \\
\hline Isla de Maipo & 0,19 \\
\hline Alhué & 0,10 \\
\hline María Pinto & 0,08 \\
\hline San Pedro & 0,07 \\
\hline
\end{tabular}

Fuente:INE, 1992 
Inmigrantes hacia el Gran Santiago

1987 - 1982

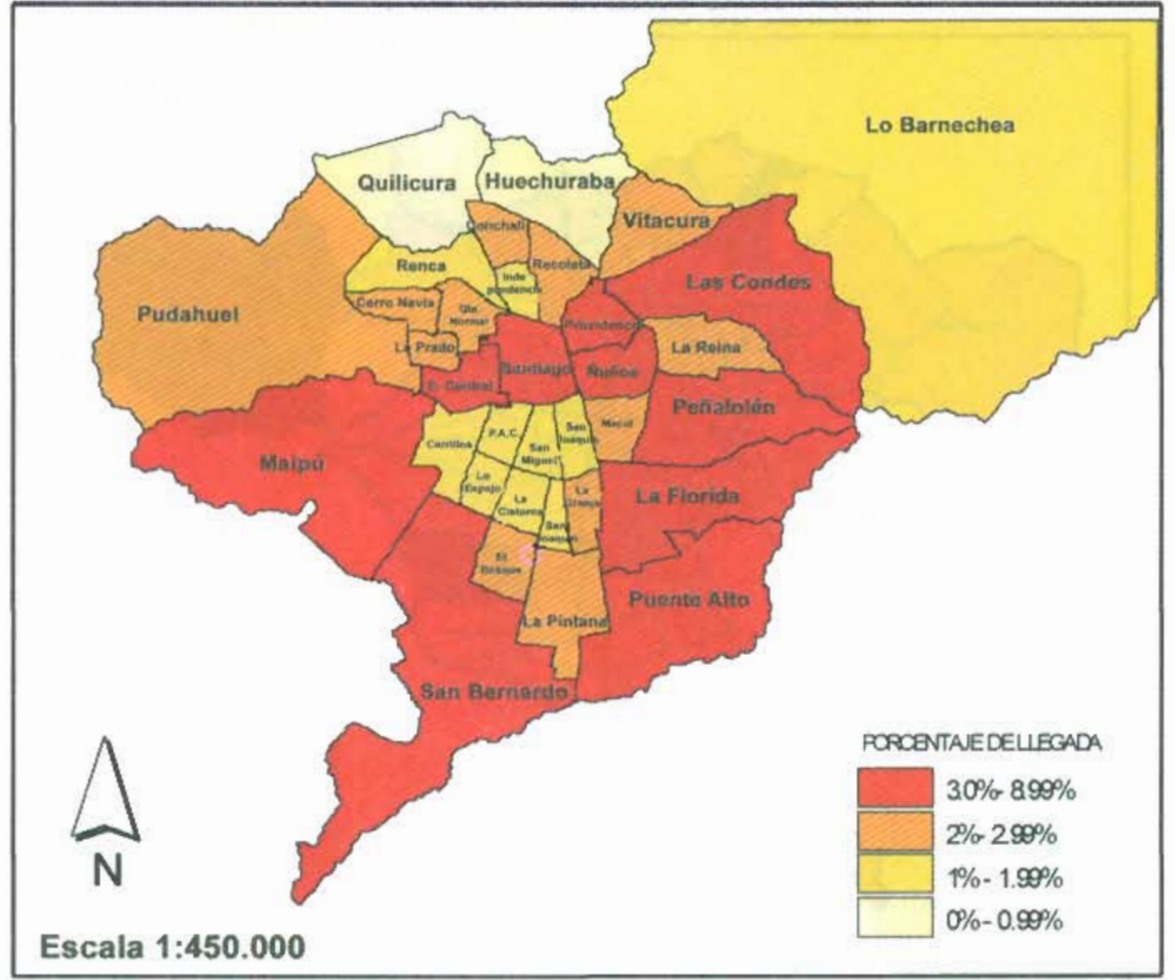

Furnte I.N.E 1998

Fig. 3. Patrón de destino de los inmigrantes extraregionales hacia el Gran Santiago. Se puede ver que tanto comunas centrales como periféricas concentran la mayor cantidad de inmigrantes.

Fig. 3. Destination pattern of extra regional immigrants to the Greater Santiago. It can be seen that both the central communes and the peripherical ones concentrate the larger proportion of immigrants.

Los resultados alcanzados de la aplicación del índice de segregación, indican que no existen distritos que ejerzan un poder de atracción superior a otros, por lo que se concluye que el patrón de distribución resulta ser más bien homogéneo. Sin embargo, de la aplicación del índice a un nivel intracomunal, se constata que existen entidades espaciales que captaron más la atención que otras. Resaltan los casos de comunas periféricas como Lo Barnechea, Puente Alto, Quilicura y de comunas interiores como La Cisterna, Estación Central y Pedro Aguirre Cerda (Figs. 3 y 4 ).

Hay que destacar que las 6 comunas que recibieron la mayor cantidad de flujos regio- nales, no manifiestan una segregación espacial en su distribución, sino más bien un comportamiento francamente homogéneo (Figs. 3 y 4 ; Tabla 4 ).

\section{Tipología del inmigrante regional}

La migración genera mucho más que una simple redistribución de población que puede y debe ser medida. Cada migrante tiene ciertos atributos: edad, sexo, status familiar, ocupación, logros educacionales, culturales, creencias religiosas, etc. Ellos han sido tratados in extenso en estudio de migraciones, tanto de tipo forzado como en movimientos espontáneos de carácter ínter e 


\section{Inmigrantes hacia el Gran Santiago \\ 1987 - 1982}

Indice de Segregación Espacial

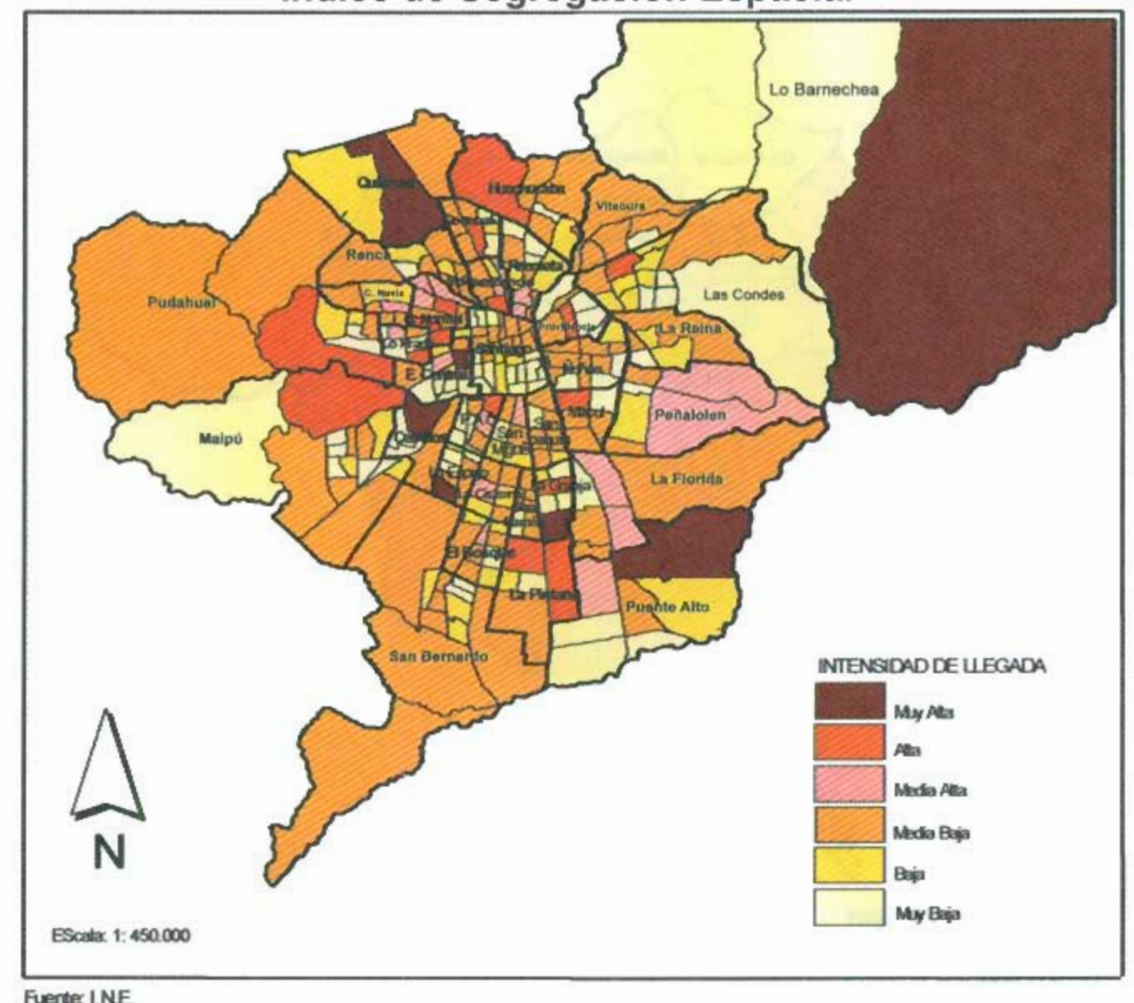

Fig. 4. Resultados de un análisis más detallado: intracomunal. Los distritos periféricos los que concentran la mayor proporción de población migrante. Fig. 4. Results in a more detailed analysis: inside the communes. The peripheral districts of the city have the highest proportion of immigrants.

intracontinental, intraurbano, regional, etc. Lo anterior, motivado fundamentalmente por la existencia de ciertos patrones que tienden a repetirse en las características asociadas al perfil del migrante, y en el destino que estos eligen al momento de optar por el nuevo lugar de residencia. Para indagar en torno a las características que asume el inmigrante en el destino, el análisis se centrará en las diez comunas que concentraron más del $50 \%$ de los movimientos, destacándose al mismo tiempo, que sus localizaciones espaciales son tanto de tipo central como periféricas, representando estos espacios status socioeconómicos diversos.

$\mathrm{Al}$ analizar el sexo, las mujeres migran más que los hombres $(53,6 \%)$, patrón que no re- sulta del todo homogéneo, si se compara con la región de origen. La Fig. 5, muestra un claro predominio femenino en distancias cortas, destacando la IX región con la de mayor proporción femenina $(57,6 \%)$. Sin embargo los hombres son los más propensos a emigrar cuando la distancia se incrementa: como es el caso de las regiones de Tarapacá $(52,0 \%)$, Aysén $(51,5 \%)$ y Magallanes $(53,4 \%)$.

En este sentido las comunas de destino que polarizan la mayor cantidad de movimientos femeninos resultan ser aquellas de un status socioeconómico superior como Las Condes, Providencia y Ñuñoa. Sólo en tres entidades dominan levemente los hombres. Los factores de esta distribución comienzan a vislum- 


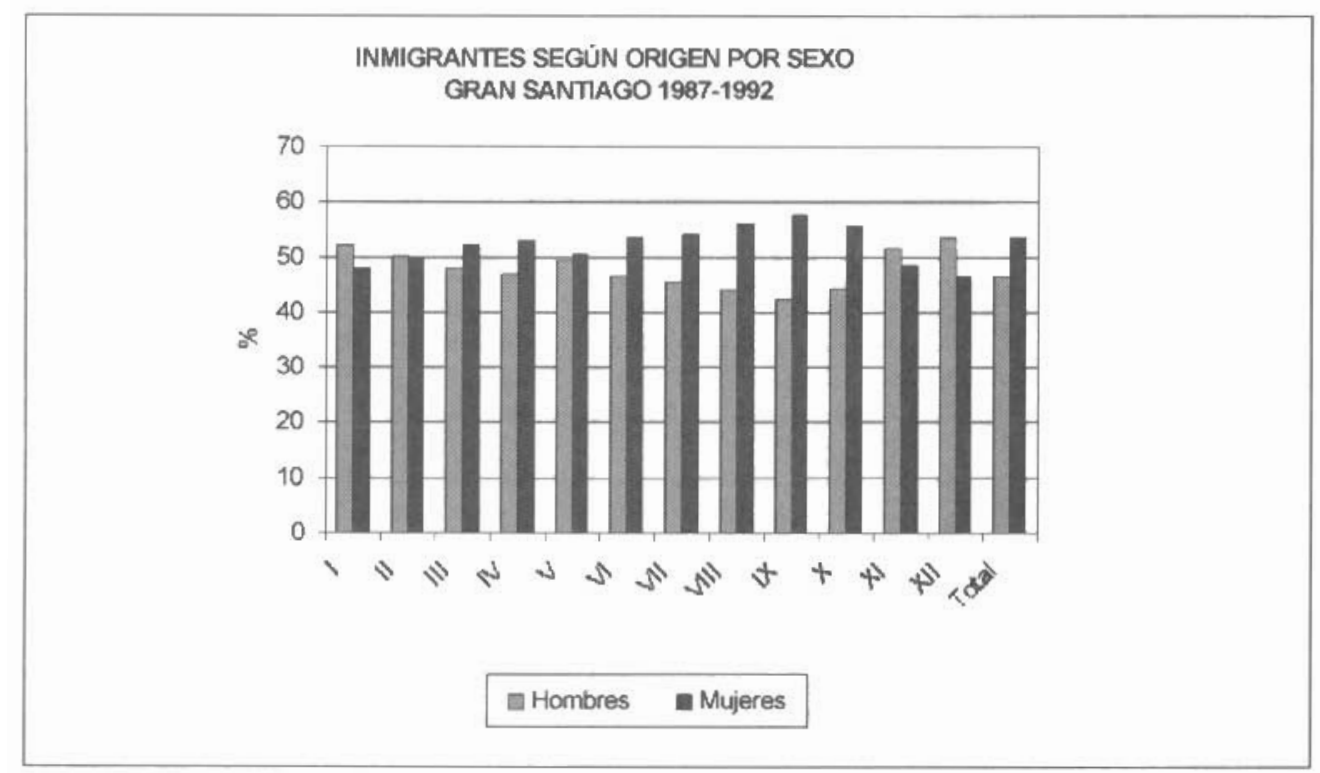

Fig. 5. La distancia, aún es un factor significativo en la interacción espacial. Aquellas regiones cercanas a Santiago aportan más mujeres inmigrantes, mientras que, en aquellas más distantes la proporción mayoritaria es de hombres.

Fig. 5. The distance is still an important factor in the spatial interaction. Regions closer to Santiago, have the main proportion of female immigrants, meanwhile in the remote ones, the largest proportions of immigrants are men.

brarse en la medida que se interpreta el resto de los atributos asociados, en particular el nivel de ocupación declarado.

El predominio de la mujer en la migración hacia áreas urbanas en Latinoamérica puede interpretarse empleando la tasa de rendimiento: en las áreas de origen de los movimientos existen pocos empleos productivos para las mujeres, mientras que las oportunidades en las grandes metrópolis son mejores. La mayoría de las mujeres, especialmente las que acaban de llegar al lugar de destino, trabajan en el servicio doméstico. A esto se debe el gran contraste que hay entre la distribución por ocupación de las mujeres que emigran y las nativas (BRIGG 1975).

La edad (Tabla 5) resulta ser el único atributo que se puede asociar efectivamente al momento en que se inició el movimiento; se recuerda que el resto de las caracte- rísticas a ser analizadas corresponden a lo que el migrante declaró cinco años más tarde. En este orden de ideas, se trata de un flujo de población preferentemente joven, puesto que el $40 \%$ de los migrantes tenía entre 15 y 24 años. Estos antecedentes hacen pensar en un predominio de la migración individual por sobre los movimientos de grupos familiares. En las diez comunas seleccionadas predomina la misma cohorte, alcanzando la mayor representatividad en el espacio central de la ciudad. Por lo tanto, en general, existe una mayor propensión a migrar por parte de la población joven adulta. Los niños tienden a concentrarse en las comunas periféricas con una baja presencia en Santiago y Providencia. El patrón de distribución de la tercera edad tiene una participación relativamente homogénea en su localización, independientemente del nivel socioeconómico de la comuna receptora. 
Tabla 5. Población inmigrante hacia el Gran Santiago por Rangos de edad Tabla 5. Immigrant population to Greater Santiago by age stages.

\begin{tabular}{|c|c|}
\hline Rangos de edad (años) & $\begin{array}{c}\% \text { del total de } \\
\text { migrantes }\end{array}$ \\
\hline $5-9$ & 5,8 \\
\hline $10-14$ & 6,5 \\
\hline $15-19$ & 13,4 \\
\hline $20-24$ & 22,2 \\
\hline $25-29$ & 16,7 \\
\hline $30-34$ & 10,8 \\
\hline $35-39$ & 6,6 \\
\hline $40-44$ & 4,2 \\
\hline $45-49$ & 3,1 \\
\hline $50-54$ & 2,5 \\
\hline $55-59$ & 1,9 \\
\hline $60-64$ & 1,9 \\
\hline $65-69$ & 1,4 \\
\hline $70 y+$ & 3 \\
\hline
\end{tabular}

Fuente: INE, 1992

El estado civil declarado 5 años más tarde (Tabla 6), resulta coincidente con lo descrito en el análisis etario, en la medida que, prácticamente el $50 \%$ de la población se declara soltera, destacando las comunas reconocidas como "más ricas», con cifras superiores al
50\%. Los casados, categoría que se puede asociar a un núcleo familiar binuclear a polinuclear, predominan en entidades periféricas - San Bernardo y Maipú- espacios donde se detecto una presencia significativa de niños.

Tabla 6. Población inmigrante hacia el Gran Santiago por Estado Civil

Table 6. Immigrant popUlation to Greater Santiago by Civil SITUation

\begin{tabular}{|c|c|}
\hline Estado civil & $\%$ \\
\hline Casado & 36,78 \\
\hline Conviviente & 6,85 \\
\hline Soltero & 47,13 \\
\hline Viudo & 5,57 \\
\hline Separado & 3,20 \\
\hline Anulado & 0,47 \\
\hline
\end{tabular}

Fuente: INE, 1992 
La situación laboral en que se encontraban los inmigrantes en 1992 (Tabla 7), aparece altamente relacionada con las características etarias descritas. Casi el $55 \%$ se declara como estudiante sin trabajar, categoría que se repite en las entidades en análisis. Bastante distante, con un $19,3 \%$, aparece la categoría «quehaceres del hogar», cuyos porcentajes más bajos se manifiestan en comunas de status superior como Las Condes y Providencia.

\section{Tabla 7. Población inmigrante hacia el Gran Santiago por situación Laboral Table 7. IMmigrant population to Greater Santiago by wORK SITUATION}

\begin{tabular}{|c|c|}
\hline Situación laboral & $\%$ \\
\hline Trabajando con ingreso & 53,7 \\
\hline Sin Trabajar & 1,9 \\
\hline Trabajando sin pago & 0,4 \\
\hline Busca trabajo, antes tenia & 3,1 \\
\hline Busca trabajo por 1ra.Vez & 0,9 \\
\hline Quehaceres del Hogar & 19,3 \\
\hline Estudia sin Trabajar & 11,9 \\
\hline Pensionado, jubilado & 4,2 \\
\hline Incapacitado Permanente & 0,6 \\
\hline Otra Situación & 3,5 \\
\hline
\end{tabular}

Fuente: INE, 1992

La condición de ocupación resulta ser una variable inportante de incorporar (Tabla 8), porque contestan esta pregunta los inmigrantes que forman parte de la población económicamente activa. El $66 \%$ de la población que emigró a Santiago entre 1987 y 1992 , se declara como trabajador asalariado, lo que permite concluir que la ciudad acoge finalmente al migrante con un trabajo que se puede tipificar como estable. Aquellos que trabajan en el servicio doméstico representan la segunda participación con un $21 \%$, con una presencia importante en las comunas de Las Condes, Providencia y Ñuñoa.

Esta eventual «acogida», puede estar asociada a los bajos montos de recepción de inmigrantes durante el período en estudio. La asimilación depende del volumen de la población del grupo inmigrante. En general, cuanto más numerosa y diferenciada es ésta, más amenazado se sentirá el grupo de acogi- da y más difícil será la asimilación (CAPEL, 1997).

Por otro lado, BRIGG (1975), encuentra que la asimilación de los que emigran a una zona urbana depende principalmente de su selectividad. BALAN (1969) examinó estos factores en relación con varias ciudades latinoamericanas, con el propósito de tratar de explicar las diferencias socioeconómicas entre migrantes y nativos. En América Latina cada vez se otorga mayor importancia a los antecedentes de una persona, como el haber terminado los estudios primarios o secundarios.

En este contexto, los antecedentes referidos al nivel educacional indican que se trata de un movimiento selectivo. En efecto el $15 \%$ posee estudios universitarios, mientras que el $46 \%$ esta cursando o finalizó la enseñanza media. Esta tipificación de selectividad, se basa en que en la actualidad esta situación 
Tabla 8. Población inmigrante hacia el Gran Santiago por ocupación Tabla 8. Immigrant population to Greater Santiago by SPECIFIC WORK

\begin{tabular}{|l|c|}
\hline \multicolumn{1}{|c|}{ Situación de Ocupación } & $\%$ \\
\hline Personal del ejecutivo y legislativo & 4,1 \\
\hline Profesionales & 8,6 \\
\hline Técnicos & 5,0 \\
\hline Ernpleados de oficina & 11,4 \\
\hline Vendedores y trabajadores del comercio & 9,6 \\
\hline Agricultores, agropecuarios y pesqueros & 1,6 \\
\hline Operarios y artesanos & 14,2 \\
\hline Operadores maquinaria & 5,8 \\
\hline Trabajadores no calificados & 35,0 \\
\hline Fuerzas armadas y de orden & 4,7 \\
\hline
\end{tabular}

Fuente: INE, 1992

podría haberse superado, considerando la edad predominante en las corrientes.

Finalmente el lugar de nacimiento de la población, se constituye en una variable útil para medir eventuales flujos cle retorno, es decir, de la población nacida en la Región Metropolitana, y que vuelve a su espacio de origen. Prácticamente el $80 \%$ de los inmigrantes nacieron en una región diferente a la de destino.

\section{Análisis macroeconómico y oferta de ser- vicios}

El análisis realizado, da cuenta de la fuerte disminución de movimientos hacia la Región Metropolitana durante el período 1987-92, identificando tres regiones como las que aportaron prácticamente el $50 \%$ de los inmigrantes: del Bío-Bío, de Valparaíso y del Maule. Por otra parte, se concluye que el comportamiento de los emigrantes es claramente selectivo respecto del lugar que escogieron como destino, sobre la base de sus características laborales, educativas, sexo, etc., situación que se refleja espacialmente, en las comunas que reciben los mayores flujos. Es previsible aventurar que en las estadísticas del último Censo (2002), se observe la tendencia a una disminución constante de flujos migratorios hacia Santiago y, por ende, una mayor selectividad de los emigrantes al momento de tomar la decisión de moverse.

Establecido el panorama general anterior, es necesario intentar encontrar causales que hayan motivado los movimientos registrados en el período censal analizado. Como este instrumento no contiene preguntas específicas referentes a motivos, hay que establecer un procedimiento metodológico que pueda llegar a determinar estas causas de manera indirecta.

El reducido número de emigrantes hacia Santiago y su selectividad, induce a identificar alguna correlación entre factores regionales, que expliquen su desplazamiento. De ahí que se haya optado en primer término por un análisis del comportamiento macroeconómico regional. Resulta conveniente destacar que este análisis se refiere exclusivamente al comportamiento de las tres regiones con mayores aportes (V, VII y VIII), comparadas con la Región Metropolitana. Por otra parte, aprovechando las series históricas disponibles para los informes macroeconómicos nacio- 
nales, en términos temporales, el análisis se extendió hasta 1985, incorporando tres años más, en la medida que la decisión de migrar no necesariamente se toma el mismo año en que se efectúa el movimiento.

El análisis se basa en que debería haber indicadores promedios del Producto Interno Bruto (PIB), que señalen que las regiones expulsoras han sufrido problemas macroeconómicos complejos en el período, cuando - simultáneamente - la Región Metropolitana debería haber crecido significativamente, provocando una fuerte atracción.

El Producto Interno Bruto (PIB) para el período 1985-1992, muestra un comportamiento de constante crecimiento, tanto nacional como regional. Durante el período, todas las regiones incrementaron sus respectivos PIB, sin embargo estos incrementos no fueron de la misma magnitud. De esta forma se destaca un crecimiento promedio muy significativo de la Región Metropolitana la que casi duplica su valor durante el período $(9.02 \%$ anual). Las tasas promedios para las regiones en estudio son: VII (7.95\%), V (6.04\%) y VIII (5\%). Es significativo señalar que estas dos últimas presentan un crecimiento que está por debajo del promedio nacional durante el período (7.39\%).

Por tanto, cabe suponer que existe una primacía de la Región Metropolitana en el crecimiento general para el período, lo que indicaría un posible factor atractivo de tipo difuso y general. Sin embargo, aún así, ella no es lo suficientemente grande como para asegurar por sí sola un movimiento migratorio importante hacia la región receptora (Tabla 9).

Tabla 9. Crecimiento anual del PIB regional (1986-1992) Table 9. AnNual growing of the Regional PIB (1986-1992)

\begin{tabular}{|c|c|c|c|c|c|c|c|c|}
\hline REGION & $1985-86$ & $1986-87$ & $1987-88$ & $1988-89$ & $1989-90$ & $1990-91$ & $1991-92$ & Promedio \\
\hline I & 4.95 & 1.96 & 2.47 & 9.86 & 0.37 & 5.97 & 13.70 & 5.72 \\
\hline II & 1.77 & -0.14 & 8.23 & 17.36 & 2.07 & 13.21 & 1.68 & 7.07 \\
\hline III & 1.05 & 9.45 & 6.25 & 11.29 & 6.69 & 18.38 & 13.50 & 10.93 \\
\hline IV & 6.11 & 1.41 & 20.21 & 6.49 & 3.84 & 6.83 & 12.64 & 8.57 \\
\hline V & 1.94 & 3.34 & 10.32 & 8.48 & 2.72 & 3.67 & 7.71 & 6.04 \\
\hline VI & 9.15 & 3.57 & 1.39 & 3.03 & 0.83 & 3.45 & 11.17 & 3.91 \\
\hline VII & 7.75 & 3.80 & 8.86 & 3.54 & 4.60 & 10.91 & 15.99 & 7.95 \\
\hline VIII & 2.73 & 4.28 & 7.56 & 3.82 & 3.48 & 3.59 & 7.28 & 5.00 \\
\hline IX & 0.14 & 12.82 & 11.43 & 6.91 & 1.29 & 3.33 & 14.14 & 8.32 \\
\hline $\mathrm{X}$ & 7.36 & 9.52 & 3.79 & 5.71 & 9.95 & 1.18 & 9.62 & 6.63 \\
\hline $\mathrm{XI}$ & 7.36 & 8.52 & 2.96 & 1.53 & 4.98 & 1.10 & 6.21 & 4.22 \\
\hline $\mathrm{XII}$ & 1.46 & 5.83 & -1.11 & 11.06 & 0.37 & 4.05 & 0.28 & 3.41 \\
\hline $\mathrm{RM}$ & 7.62 & 8.13 & 6.85 & 11.53 & 3.79 & 9.66 & 14.18 & 9.02 \\
\hline PAIS & 5.40 & 5.88 & 6.99 & 9.29 & 3.42 & 7.63 & 11.15 & 7.39 \\
\hline
\end{tabular}

Fuente: Ministerio de Economía, 1998. 
Este análisis, por sí solo no es concluyente, ni permite establecer patrones que apunten hacia comportamientos modélicos. Por ello, se hace necesario agregar otro elemento: el comportamiento evolutivo regional respecto del PIB a un nivel de resolución menor, es decir, la evolución regional por rama de actividad económica, en la medida que resulta necesario comprender la interrelación de los componentes de mayor importancia en los PIB regionales, comparados con los mismos en la Región Metropolitana. El resultado incide en el componente de selectividad que se ha detectado en los movimientos migratorios, ya que éstos no coinciden necesariamente con las demandas desde la Región Metropolitana por ciertas funciones, para las que históricamente las regiones han otorgado oportunidades. Esto invalida la hipótesis de movimientos basados en la búsqueda de oportunidades de empleo, que se daría en el caso de haber descendido bruscamente el factor en la región expulsora. Se complementa entonces el análisis con una visión de las tasas de desempleo regionales en el período.

Para evitar un análisis exhaustivo que involucre largas tablas comparativas, es posible indicar resumidamente que se detectan para la Región Metropolitana los siguientes sectores ${ }^{1}$ como los de mayor dinamismo: Industria manufacturera, crece de $247.094 \mathrm{mi}$ llones de pesos anuales ${ }^{2}$, a 459.243 en 1992. Sector Comercio, restaurantes y hoteles, de 297.814 a 602.077 millones anuales. Servicios Financieros, de 308.333 a 572.773 millones anuales. En conjunto corresponden al 75\% del PIB regional. Claramente, la Región Metropolitana incrementa en el período su participación en servicios terciarios, y gran parte de su crecimiento promedio, analizado anteriormente, se deriva hacia estos sectores. En el caso de las regiones V, VII y VIII, su comportamiento en los mismos sectores es el siguiente:

- Industria: V región, 77.863 a 85.563 millones anuales, crecimiento moderado- bajo; VII región, de 26.041 a 39.364 millones, crecimiento medio; VIII región, de 125.598 a 169.556 millones, crecimiento casi nulo, pero aún así con índices positivos.

- Comercio: V región, de 32.415 a 51.456, crecimiento moderado; VII región, 10.006 a 19.461 millones, a pesar del bajo impacto sobre el PIB regional crece casi al doble; VIII región, 28600 a 41,731 millones, crecimiento acelerado.

- Financieros: V región, 17.435 a 30.453 millones, considerado como acelerado; VII región, de 6.708 pasa a 12.728 millones, evidenciando la aparición de un sistema financiero de creciente importancia relativa; VIII región, de 13.920 a 31.658 millones, también un crecimiento acelerado, pero que aún refleja la vocación regional vertida hacia la industria y la producción, como componentes principales del PIB regional.

Cabe señalar que, durante la década de los 80, como política de Estado, se incrementa la inversión y la producción en las actividades ligadas a la exportación de materias primas, en especial las derivadas de la fruticultura. En este sentido, la VII región pudo mantener su aporte al PIB, generando un crecimiento de 33.543 a 65.968 millones de pesos, que complementa el crecimiento moderado de los otros sectores y arroja una tasa positiva final para la región en el período. Por otra parte, aún cuando las regiones $\mathrm{V}$ y VIII siguen creciendo en el período en términos absolutos, las políticas que se aplican en ellas, y la participación del sector privado, apuntan hacia el impulso de la agro industria, la que frente a crisis mundiales ve afectada su participación. Esta situación se refleja en una disminución en su contribución sectorial al PIB nacional.

En resumen, desde el punto de vista del PIB destaca la Región Metropolitana, como un área de gran crecimiento económico en el período. La VII región aparece como un área

1 El análisis sectorial se realizó para el período 1985-1992, de acuerdo con los Anuarios del Banco Central de Chile para esos años. 
de crecimiento medio, mientras que las regiones V y, sobre todo la VIII, muestran crecimientos moderados, aunque en ningún caso decrecimiento.

Ante esta constatación, no resulta paradójico que el flujo total de emigrantes aparezca reducido, ya que - aún cuando la Región Metropolitana mantiene una fuerte capacidad competitiva y atractriz específica- las regiones expulsoras no se encontraban en crisis económicas globales, ni manifestaban profundas diferencias entre los factores señalados. Solo en la VII región del Maule, se puede identificar un problema derivado del estancamiento del crecimiento industrial en el período. Sin embargo el perfil del migrante no señala una fuerte preponderancia de mano de obra industrial migrante.

Se hace necesario entonces, incorporar nuevos antecedentes complementarios de análisis: la Población Económicamente Activa
(PEA) regional y las tasas de desempleo regionales. Esta última variable, según RACZYNSKI (1978), permite incorporar la dinámica de la generación de empleo en un área, análisis complementario al de las tasas de desocupación.

Para el período 1986-1991, la PEA, presenta un crecimiento moderado en la Región Metropolitana, de 3,98\% (valor superior al nacional) ampliamente superada por la VII y VIII región $(4,28 \%)$ y sin diferir significativamente de la Región de Valparaíso (Tabla 10).

De esta forma se puede afirmar que el crecimiento de la PEA no entrega elementos discriminadores para afirmar grandes diferencias en la base económica de las regiones analizadas. Para reafirmar lo anterior, se analizan las proporciones de participación regional en la PEA nacional, donde todas las regiones mantienen su participación porcentual (Tabla 11).

Tabla 10. Crecimiento anual PEA regional (1986-1991)

Table 10. Annual growing of the regional PEA (1986-1991)

\begin{tabular}{|c|c|c|c|c|c|c|}
\hline REGION & $1986-87$ & $1987-88$ & $1988-89$ & $1989-90$ & $1990-91$ & Promedio \\
\hline I & 6,62 & 2,02 & 3,22 & 3,59 & 9,99 & 5,09 \\
\hline II & 6,50 & $-0,06$ & 4,58 & 3,85 & 1,26 & 3,23 \\
\hline III & 6,08 & 5,61 & 7,26 & $-0,66$ & 1,56 & 3,97 \\
\hline V & 5,81 & 5,91 & 3,31 & 3,05 & 4,27 & 4,47 \\
\hline VI & 1,79 & 6,63 & 4,27 & 0,84 & 4,82 & 3,67 \\
\hline VII & 0,92 & 6,53 & 0,56 & 2,20 & 2,51 & 2,54 \\
\hline VIII & 1,98 & 2,56 & 4,90 & 3,57 & 3,68 & 4,28 \\
\hline IX & 4,19 & 6,92 & 2,22 & 1,31 & 2,08 & 3,34 \\
\hline$X$ & 3,97 & 5,36 & 4,92 & 2,25 & 3,34 & 3,97 \\
\hline$X I$ & 6,03 & 3,87 & 1,90 & 1,94 & 0,70 & 2,89 \\
\hline$X I I$ & 0,16 & 3,21 & 4,10 & $-0,23$ & $-3,92$ & 0,66 \\
\hline RM & 4,44 & 6,26 & 7,24 & 2,65 & $-0,72$ & 3,98 \\
\hline Total & 3,78 & 5,90 & 5,54 & 2,15 & 1,58 & 3,79 \\
\hline
\end{tabular}

Fuente: Ministerio de Economía, 1998. 
Tabla 11. Participación regional sobre la PEA nacional $(\%), 1985-1991$

Table 11. Regional participation On the national PEA (\%), 1985-1991

\begin{tabular}{|c|c|c|c|c|c|c|}
\hline REGION & 1986 & 1987 & 1988 & 1989 & 1990 & 1991 \\
\hline I & 2,54 & 2,61 & 2,52 & 2,46 & 2,50 & 2,70 \\
\hline III & 2,89 & 2,97 & 2,80 & 2,77 & 2,82 & 2,81 \\
\hline IV & 1,55 & 1,58 & 1,58 & 1,60 & 1,56 & 1,56 \\
\hline V & 3,41 & 3,48 & 3,48 & 3,41 & 3,44 & 3,53 \\
\hline VI & 10,40 & 10,20 & 10,27 & 10,15 & 10,02 & 10,34 \\
\hline VII & 5,37 & 5,22 & 5,25 & 5,01 & 5,01 & 5,05 \\
\hline VIII & 12,37 & 12,33 & 12,59 & 12,74 & 12,58 & 12,65 \\
\hline IX & 5,74 & 5,76 & 5,81 & 5,63 & 5,58 & 5,61 \\
\hline X & 6,82 & 6,83 & 6,80 & 6,76 & 6,76 & 6,88 \\
\hline XI & 0,69 & 0,71 & 0,70 & 0,67 & 0,67 & 0,66 \\
\hline XII & 1,34 & 1,30 & 1,26 & 1,25 & 1,22 & 1,15 \\
\hline RM & 40,12 & 40,37 & 40,51 & 41,17 & 41,37 & 40,43 \\
\hline Tota! & 100,00 & 100,00 & 100,00 & 100,00 & 100,00 & 100,00 \\
\hline
\end{tabular}

Fuente: Ministerio de Economia, 1998.

La tercera variable analizada corresponde a la tasa de desempleo, indicador que muestra el comportamiento más contradictorio para explicar los flujos migratorios hacia el Gran Santiago. Durante gran parte del período 1986-1991 la tasa promedio de desocupación de la Región Metropolitana fue claramente superior a la de las restantes regiones. Sin embargo, al realizar un análisis algo más detallado, se puede afirmar que el decrecimiento del desempleo, y la capacidad de generar empleo, son muy significativos en la Región Metropolitana, tanto así que, durante 1991, casi igualaba la tasa de las restantes regiones. De esta forma, el desempleo decreció en esta región a una tasa del orden del $-8.7 \%$ anual, a un ritmo bastante superior a las regiones V $(-5.9 \%)$, VII $(-0.5 \%)$ y VIII $(2.4 \%)$ (Tabla 12).

Finalmente, y en la medida que la tipología del inmigrante demostró un predominio de la población joven, entre 15 y 24 años, se incorpora al análisis antecedentes sobre matrícula total de educación superior para el período 1983-1991. Las cifras muestran un significativo incremento de cupos en la Región Metropolitana, la que incrementa su participación en el total nacional desde un $48,5 \%$ a una cifra superior al $50 \%$. Este antecedente, la convierte en un área de alta concentración de oferta educacional (Tabla 13). En este mismo período, las regiones V y VIII presentaron crecimientos relativamente moderados y la VII región mantuvo su proporción y presentó ciertos períodos de disminución.

La ciudad se constituye entonces en el lugar de la movilidad social, del ascenso social. Los concluyentes datos, permiten demostrar que las posibilidades que ofrece la metrópolis principal son mayores que aquellas que se presentan en regiones. Los testimonios de ello 
Tabla 12. Crecimiento anual Tasa de Desocupación Anual, 1986-1991

Table 12. Annual growing of Annual Unemployment Rate, 1986-1991

\begin{tabular}{|c|c|c|c|c|c|}
\hline Región & Pais & Valparaiso & Maule & Bio-Bio & Santiago \\
\hline $1986-87$ & $-9,8$ & $-21,1$ & $-12,1$ & 7,7 & $-7,8$ \\
\hline $1987-88$ & $-10,8$ & $-6,8$ & 4,6 & $-6,7$ & $-12,1$ \\
\hline $1988-89$ & $-18,8$ & $-19,4$ & $-5,6$ & $-15,1$ & $-24,6$ \\
\hline $1989-90$ & $-1,5$ & 18,6 & 6,7 & $-4,5$ & $-6,3$ \\
\hline $1990-91$ & 4,7 & $-0,7$ & 4,1 & 6,6 & 7,2 \\
\hline Promedio & 7,2 & $-5,9$ & $-0,5$ & $-2,4$ & $-8,7$ \\
\hline
\end{tabular}

Fuente: INE, 1999.

son abrumadores en ciudades de diferentes épocas históricas y de diferentes países (CAPEL, 1997).

Las cifras demuestran el centralismo y la concentración de la capacidad científico - tecnológica en la Región Metropolitana. Según GONZÁLEZ (1996), de 1.725 doctores contabilizados en el país hasta el año 1994 en las Universidades del Consejo de Rectores, el $52,1 \%$ correspondían a la Región Metropolitana, y un $47,9 \%$ a regiones. La VIII Región aparece en un segundo lugar con solo el $14,8 \%$.

Se desprende que existe una fuerte tendencia, culturalmente enraizada, a considerar de alta calidad sólo aquellos servicios y capacidades profesionales existentes en la capital del país. La consecuencia es suponer que en las regiones por principio existe un servicio de baja calidad y bajo nivel profesional, sin que ello se fundamente necesariamente sobre la base de criterios objetivos (VON BAER, 1996)

Dado que el análisis de variables macroeconómicas, de empleo y evolución de las matrículas en la educación superior, no entrega antecedentes explicativos concretos de estos movimientos, tanto desde el punto de vista de la expulsión como de factores atractivos de la Región Metropolitana sobre las regiones de origen, es importante introducir otro campo de análisis en la discusión, que permita contrastar lo revisado anteriormente.

$\mathrm{Al}$ respecto, los resultados regionales y nacionales sobre el Índice de Desarrollo Humano (IDH) ${ }^{3}$ para Latinoamérica y Chile (PROGRAMANACIONES UNIDAS PARA EL DESARROLLO, PNUD-COMISIÓN ECONÓMICA PARA AMÉRICA LATINA, CEPAL en 1996) ${ }^{4}$, se contrastan con los entregados por el Índice de Desarrollo Humano en las Comunas de Chile (PNUD-MINISTERIO DE PLANIFICACIÓN, MIDEPLAN, 2000), puesto que es el único estudio de nivel territorial desagregado de la última década en esta materia. Chile se sitúa en el lugar 33 de la clasificación mundial respecto del IDH. Esto indica que el crecimiento económico chileno en los últimos años, se traduce en beneficios para las personas marcando un proceso de acumulación histórico - cultural de potencialidades de la sociedad chilena. Sin embargo, el país presenta un con-

3 Metodología y contenidos pueden ser consultados en www.desarrollo humano.cl

4 Si bien el estudio de Naciones Unidas es de 1996, su espectro abarca desde 1982 a 1995, por lo que queda dentro de los marcos temporales utilizados por el censo de 1992, al consultar acerca de la residencia del encuestado en 1987. 
Tabla 13. Participación regional en Matrícula Total de Educación Superior 1983-1992 (\%)

Table 13. Regional participation in Total Register of Highest Education, 1983-1992 (\%)

\begin{tabular}{|c|c|c|c|c|c|c|c|c|}
\hline REGION & 1983 & 1985 & 1986 & 1987 & 1989 & 1990 & 1991 & 1992 \\
\hline I & 3.5 & 3.6 & 3.4 & 3.4 & 3.0 & 3.0 & 3.6 & 3.3 \\
\hline II & 4.7 & 4.0 & 4.6 & 3.5 & 3.3 & 3.7 & 3.2 & 3.8 \\
\hline III & 1.0 & 1.1 & 1.0 & 1.0 & 0.8 & 0.9 & 0.9 & 0.8 \\
\hline IV & 3.2 & 3.2 & 3.0 & 3.1 & 3.0 & 2.7 & 2.6 & 3.1 \\
\hline V & 12.1 & 12.2 & 12.3 & 11.9 & 12.3 & 12.3 & 12.4 & 11.9 \\
\hline VI & 0.4 & 0.5 & 0.6 & 0.8 & 1.0 & 1.2 & 1.3 & 1.2 \\
\hline VII & 3.4 & 3.4 & 3.4 & 3.5 & 3.3 & 2.4 & 2.9 & 2.7 \\
\hline VIII & 12.4 & 13.4 & 13.4 & 13.3 & 13.1 & 13.1 & 14.0 & 13.6 \\
\hline IX & 4.2 & 4.2 & 3.8 & 3.8 & 3.9 & 3.8 & 3.7 & 3.8 \\
\hline $\mathrm{X}$ & 5.8 & 5.4 & 5.6 & 5.6 & 5.4 & 5.0 & 5.4 & 5.1 \\
\hline $\mathrm{XI}$ & 0.0 & 0.1 & 0.1 & 0.1 & 0.0 & 0.0 & 0.0 & 0.0 \\
\hline $\mathrm{XII}$ & 0.9 & 0.9 & 0.8 & 0.9 & 0.7 & 0.7 & 0.8 & 0.7 \\
\hline $\mathrm{RM}$ & 48.5 & 48.2 & 48.2 & 49.1 & 50.3 & 51.1 & 49.3 & 50.2 \\
\hline PAIS & 100.0 & 100.0 & 100.0 & 100.0 & 100.0 & 100.0 & 100.0 & 100.0 \\
\hline
\end{tabular}

Fuente: Ministerio de Educación, 2000.

junto de importantes vulnerabilidades que de alguna manera, están relacionadas con motivaciones de las personas a cambiar de región de residencia, justificando de manera general, la migración selectiva hacia la Región Metropolitana de Santiago.

Las principales vulnerabilidades tienen que ver con la distribución del ingreso. En general el país mantiene el mismo patrón distributivo que tenía en la década de los sesenta. En efecto la relación de participación en los ingresos del $10 \%$ más rico, respecto del $40 \%$ más pobre es de 3 a 1 . Esto indica una situación altamente regresiva, peor que la de otros países latinoamericanos como es el caso de Argentina (2,1 a 1), Costa Rica (1,6 a 1), Uruguay $(1,2$ a 1$)$ y México (2,1 a 1$)$, según CEPAL (1996).
Otro aspecto complejo es el de una enorme falta de equidad en cuanto las oportunidades y participación de la mujer. Este factor cobra gran importancia, considerando la alta proporción de mujeres que participan en los desplazamientos internos. En efecto, entre 1990 y 1996, el país desciende 15 lugares en la clasificación internacional (CEPAL, 1996), en relación con las disparidades entre sexos (materia en la cual solo es superado por Venezuela y Panamá).

En torno a la equidad espacial interna nacional, tanto regional como comunal, es relevante hacer un breve análisis de los resultados de este estudio con miras a buscar una posible fuente explicativa de los movimientos migratorios hacia la metrópolis. 
La Región Metropolitana se desplaza entre rangos altos y muy alto de IDH, cuya distinción se sustenta en el origen urbano o rural de la entidad. Estas últimas tienen logros calificados como medianos a bajos en el IDH, especialmente en educación, e ingresos por familia. En general, las principales comunas receptoras de inmigrantes (Tabla 4), presentan un IDH calificado como alto - muy alto en todas sus dimensiones, lo que configura «un grado de concentración de las oportunidades para el desarrollo humano, tanto a escala regional como nacional» (CEPAL 1996: 79).

Por su parte, la V Región de Valparaíso, está en el 4 lugar nacional del IDH, con logros equilibrados en salud y educación, con un importante rezago en los ingresos. El 95,5\% de la población vive en comunas de nivel alto y muy alto de IDH. Escapan a esta tendencia las comunas rurales del interior de la región, hacia su límite norte. La conurbación Valparaíso-Viña del Mar-Quilpué-Villa Alemana tiene el mejor comportamiento global de IDH, el que se distribuye homogéneamente en ellas.

La VII Región del Maule, compuesta por 30 comunas, tiene el lugar mas bajo del IDH regional nacional, con la excepción del factor salud, donde ocupa el lugar 11 del ranking. Especialmente llama la atención el bajop nivel de ingresos.Las comunas del secano costero tienen los niveles más bajos del país. Solo escapan a esta situación, las comunas de Talca y Curicó, que concentran al $33,8 \%$ de la población, y que son paradojalmente - las principales fuentes de flujos inmigratorios hacia la Región Metropolitana.

En cuanto a la región VIII, del Bío-Bío, la segunda mas poblada del país con 52 comunas, está en el 10 lugar en el ranking nacional de $\mathrm{IDH}$, ocupando el lugar trece en salud. Las zonas mas características son las agrupaciones comunales del secano costero en la provincia de Nuble con índices bajos y muy bajos. Otra agrupación similar es la del área del carbón en el sector de Arauco (Lota,
Coronel) fuertemente afectadas por la crisis de la minería y la reconversión de actividades productivas. De las 52 comunas, solo Chillán y Concepción tienen un IDH muy alto, concentrando casi al $30 \%$ de la población; nuevamente estas resultan ser las comunas más expulsoras de la región.

En resumen, los resultados del IDH para Chile indican que ha existido un notable mejoramiento, en lo referente a un desarrollo espacial regional más homogéneo en el país, a pesar de darse grandes diferencias e importantes fluctuaciones intraregionales.

\section{DISCUSION}

Al investigar las causas asociadas al patrón de movilidad interno nacional, se debiera agregar al concepto de fuerza el de inercia. De esta forma resulta mejor plantear que un grupo social en reposo, o en movimiento, tiende a permanecer en ese estado a menos que se lo impulse a cambiar; junto con cualquier patrón posible de vida se desarrolla un sistema de valores acorde con éste.

Analizar la migración interna en Chile, por ejemplo en términos de impulso y atracción, resulta totalmente inadecuado. Tal vez el problema básico debería centrarse no en por qué migra la población, sino más bien porqué no lo hace. En otras palabras ¿porqué en el mismo período (1987-1992) se registran sólo 200.000 movimientos hacia la Región Metropolitana, sobre una población de más de 8 millones que habitaba en el resto del país?.

Los factores de impulso, que se supone «causan» la emigración, por lo común comprenden un conjunto bastante heterogéneo, desde crisis agrícolas hasta el espíritu aventurero, desde la difusión de la innovación hasta el impacto de nuevas tecnologías. Pocas veces es posible establecer - y esta no es una de ellas - una diferencia entre las causas subyacentes, las facilidades del medio, los precipitantes y la motivación. Si no se establece una diferencia entre la motivación y las causas asociadas, es decir, el nivel de aspira- 
ción de los emigrantes, el análisis no posee una claridad lógica. En otsas palabras, si se hubiese podido incluir en esta investigación no sólo la causa específica asociada al movimiento, sino también las aspiraciones de quienes lo efectuaron, se podría haber contribuido a la constitución de las bases para una tipología perfeccionada de la migración interregional. Pero el Censo Nacional de población no proporciona estos antecedentes.

A pesar de lo anterior, el patrón de los movimientos analizados desde el punto de vista de su origen y características particulares, indica una situación como la descrita hace casi tres décadas por FRIEDMANN (1972). En otras palabras, el país parece encontrarse en la segunda etapa del proceso de «inigualdad» regional, caracterizado por un período de estabilidad y alto nivel de desarrollo con desigualdades espaciales en el patrón de asentamientos, manteniéndose la estructura primada, asociada a una consolidación de las llamadas ciudades intermedias. En esta etapa, se verifica una disminución sustantiva de migraciones interregionales campo-ciudad, manifestándose un flujo ciudad-ciudad como el principal, especialmente el de ciudades intermedias a la ciudad primada. En este contexto, FRIEDMANN (1972) distingue tres factores de las migraciones, que son característicos de esta etapa y que se manifiestan altamente coincidentes con los resultados alcanzados:

- Búsqueda de oportunidades de empleo, o de mejorar el existente. Este factor tiene una alta correlación con los correspondientes a educación y posibilidad de mejoramiento social de los individuos.

- Accesibilidad; al ser una función lineal de la distancia entre puntos, se verifica que la mayoría de los movimientos son desde distancias cortas y medias.

- Nivel educacional de los migrantes.

En Chile, así como en otros países latinoamericanos, los movimientos migratorios interregionales, de ciudades intermedias a la ciudad o ciudades primadas, tienen una fuerte característica de permanencia, recordando que el período considerado es de cinco años. Por otra parte, los migrantes se caracterizan por ser selectivos. Esto indica que un porcentaje significativo de ellos tiene un nivel educacional medio a alto. Los migrantes logran relocalizarse sin sufrir ajustes traumáticos en su nuevo entorno. Es decir son migrantes con capacidad de adaptación, ajuste e integración social, tal como se comprobó al indagar en torno a la situación laboral y a la condición de ocupación.

Por lo tanto, es dable concluir que los factores macroeconómicos por si solos no permiten llegar a una explicación de las causas de estos movimientos, pero que, en conjunto con lo analizado en otros estudios (CEPAL, 1996), acercan a un modelo de comportamiento ya analizado para Latinoamérica y sistematizado en un paradigma de la urbanización. En otras palabras, la explicación de los movimientos hacia la Región Metropolitana, no se encuentra en las regiones de origen, sino más bien en un conjunto de características que hacen que aquella tenga una capacidad de atracción difusa pero sistemáticamente permanente.

Esta fuerza atractriz difusa se puede debilitar al pasar a la tercera fase del modelo de FRIEDMANN (1972): la etapa de igualdad de oportunidades, al desarrollarse balanceadamente el territorio en su estructura regional interna.

\section{CONCLUSIONES}

En definitiva, se puede concluir, que la Región Metropolitana paulatinamente ha perdido peso en su rol histórico, polarizador de movimientos, incorporándose a la interpretación de esta transformación una nueva modalidad en la estructuración del sistema urbano nacional: el dinamismo experimentado por las ciudades intermedias. Este, no sólo se expresa en una consolidación de su crecimiento demográfico, sino en una oferta de «oportunidades intervinientes» (STOUFFER 1940), aportando nuevos fac- 
tores de localización que favorecen la competitividad regional: servicios avanzados a la producción, capital humano y conocimiento, además de una variada oferta de servicios públicos y privados.

Existen dos teorías que indican tendencias de crecimiento claramente diferentes para áreas metropolitanas (FREY 1988): la de la reestructuración regional y la de la desconcentración. Los resultados alcanzados permiten confirmar la hipótesis de la reestructuración en el caso del Gran Santiago. Es decir, continuará desarrollándose la tendencia a la aglomeración, pero en el marco de una nueva jerarquía funcional. Al contrario, la hipótesis de la desconcentración se aplica preferentemente en los países desarrollados; prevé un gradual, aunque sostenido despoblamiento de las áreas metropolitanas.

Se consolida en la metrópolis una nueva forma de movilidad al comioenzo de la década de los 90, con migraciones intraurbanas. Los traslados residenciales dentro de las grandes ciudades operan en términos de orientación y selectividad como factores de diferenciación espacial y socioeconómica. Este tipo de movilidad en la actualidad es la principal fuerza demográfica que incide en la segmentación socio-espacial de las ciudades (ORTIZ \& SCHIAPPACASSE 1997).

Es necesario destacar que el Censo Nacional de Población y Vivienda no registra movimientos temporales de población, que pueden significar desplazamientos geográficos, pero no cambios residenciales definitivos. En un país como Chile con migraciones estacionales, particularmente en la agricultura y silvicultura, estos elementos se constituyen en una limitación importante para el análisis integral de las migraciones.

Finalmente, resulta necesario destacar que la nueva fase de desarrollo económico que esta experimentando América Latina a comienzos de este nuevo milenio, se produce en un contexto diferente al de décadas precedentes, con profundas y desconocidas transfor- maciones económicas y sociales. Esta reestructuración de la economía latinoamericana, afecta la oferta y demanda de flujos migratorios. Estos factores deben tomarse en cuenta en políticas de regulación geográfica de las migraciones y de optimización de la distribución de la población.

\section{AGRADECIMIENTOS}

Los autores agradecen al Prof. Robert Gwynne, de la Universidad de Birmingham, la revisión crítica del manuscrito; y al Editor, su asesoría en la puesta en forma de la versión final.

\section{REFERENCIAS}

ARÉVALO, J., 1985. Problemas de la medición de la migración interna. En: Centro Latinoamericano de Demografía (Ed.), Taller de Análisis y Evaluación de los Censos de Población del 80. CELADE, Buenos Aires, pp.25-37.

BALAN, J., 1969. Diferencias socioeconómicas de emigrantes y nativos en las ciudades latinoamericanas: un análisis estructural. Latin Amer. Res. R., 4 (1): 3-29.

BRIGG, P., 1975. Migración a las áreas urbanas. En: Centro Latinoeamericano de Ciencias Sociales (Ed.), Informe del Grupo de Trabajo sobre Migraciones Internas de la Comisión de Población y Desarrollo. CLACSO, Santiago, pp: 128-191.

CAPEL, H., 1997. Los inmigrantes en la ciudad. Crecimiento económico, innovación y conflicto social. R. Electrónica de Geogr.y C.C. Soc.Scripta Nova, 3: 1-22.

CELADE, 1991. Informe sobre la situación de la población en Chile. Comisión Económica para América Latina, Naciones Unidas. LC/DEM/G.107. Serie A N²18, Santiago.

CELADE, 1999. La migración interna y sus efectos en dieciseis ciudades de Chile. Co- 
misión Económica para América Latina, Naciones Unidas LC/DEM/R.302, Santiago.

CEPAL, 1996. Informe sobre desarrollo humano (IDH). Programa de las Naciones Unidas para el Desarrollo. Santiago de Chile.

COURGEAU, D., 1986. Hyphothèse d'une transition dans le mobilité, simultanée á la transition démographique dans le monde contemporaine en développement. J. Démograph., 2: 325-331.

ELIZAGA, J.C., 1970. Migraciones a las áreas metropolitanas de América Latina. CELADE, Santiago de Chile.

FREY, W., 1988. Migración y Despoblamiento de las Metrópolis: ¿Reestructuración regional o renacimiento rural?. R. Estudios Territoriales, 28: 15-37.

FRIEDMANN, J., 1972. A general theory of polarized development. In:. Hansen, N. (Ed.), Growth centers in Regional Economic development, pp 82-107.

GONZALEZ, S., 1996. Las cifras exigen un cambio decisivo. En: Ministerio de Educación, Ministerio del Interior \& Ministerio de Planificación y Cooperación, Chile (Eds.), Universidades y Gobiernos Regionales. Actas del II Encuentro. Santiago, pp. 125-130.

ILPES, 2000. La Estructuración de los espacios regionales. Naciones Unidas. Serie Gestión Pública, 7. ILPES, Santiago.

INE, 1992. XVI Censo Nacional de Población y V de Vivienda. Instituto Nacional de Estadísticas, Santiago..

INE, 1999. Series Empalmadas de Empleo 1984-1995. Base Encuesta Nacional de Desempleo. Formato digital. Inédito.

INE, 2002. www.ine.cl, resultados preliminares XVII Censo Nacional de Población y VI de Vivienda. Información digital. Inédita.
INSTITUTO DE PATOLOGIA SOCIAL, 1968. Migración Interna hacia Santiago de Chile. Facultad de Filosofía y Educación, Universidad de Chile, Santiago.

MINISTERIO DE ECONOMIA, CHILE, 1998. Cuentas Nacionales: Informe Interno. División de Estudios. Departamento de Cuentas Nacionales, Santiago. Inédito.

MINISTERIO DE EDUCACION, CHILE, 2000. Compendio Estadístico de Educación Superior: Informe Interno, Santiago. Inédito.

PASSARIS, C., 1989. Immigration and the evolution of economic theory. Internat. Migration, 27 (4): 525-542.

ORTIZ, J. \& P. SCHIAPPACASSE, 1997. Las migraciones intraurbanas y su impacto en la diferenciación socioespacial del Gran Santiago. R. Geogr. de Chile, Terra Australis, 42: 121-138.

PNUD - MIDEPLAN, 2000. Desarrollo Humano en las Comunas de Chile. Programa Naciones Unidas para el Desarrollo, Naciones Unidas, Santiago.

PUYOL, R., 1993. Los grandes problemas actuales de la población. Colección Espacios y Sociedades, Editorial Síntesis. Serie General, 8, Madrid.

RACZYNSKI, D., 1978. Empleo, pobreza y migraciones en Chile. Colección Estudios Corporación de Investigaciones Económicas para Latinoamérica. Santiago, 29: 5-51.

RAVENSTEIN, E., 1889. The Laws of Migration. J. Royal Statist. Soc., 48: 241-301.

RODRIGUEZ, J. \& M.VILLA, 1998. Distribución espacial de la población, urbanización y ciudades intermedias: Hechos en su contexto. En: Ciudades Intermedias de América Latina y el Caribe: propuestas para la gestión urbana. CEPAL ,(LC/L.1117), Santiago de Chile, pp.26-68. 
SASSONE, S., 1991. Bases teóricometodológicas para el estudio geográfico de las migraciones. R. Argentina de Geogr., 1: 105-145.

SCHIAPPACASSE, P., 1998. Diferenciación del espacio social intraurbano en el Gran Santiago: Un análisis a nivel distrital. Tesis para optar al grado de Magister en Geografía. Facultad de Arquitectura y Urbanismo, Universidad de Chile, Santiago. Inédita.
STOUFFER, S., 1940. Intervening Opportunities: A Theory Relating Mobility and Distance, Amer. Sociol. R., 5(6): 845867.

VON BAER, H., 1996. Las Universidades, el sector productivo y el desarrollo estratégico regional. En: Ministerio de Educación, Ministerio del Interior \& Ministerio de Planificación y Cooperación, Chile (Eds.), Universidades y Gobiernos Regionales. Actas del II Encuentro. Santiago, pp. 83-96. 
Esquema de Imrestugaclones GEOBnIfitas
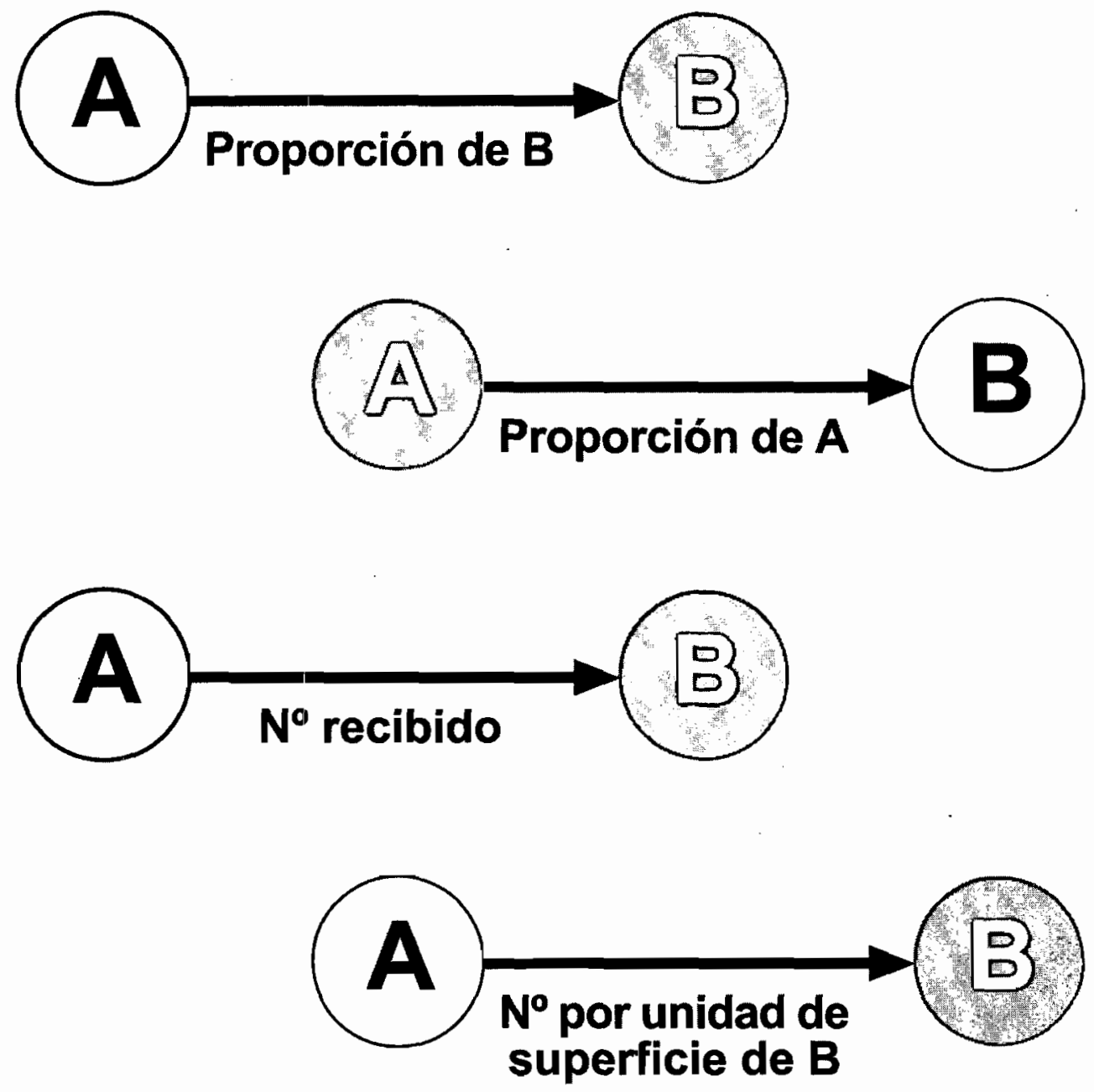

Principios para la resolución coroplética del puzzle de las migraciones. Modelo deducido a partir de los conceptos expuestos por Monkhouse \& Wilkinson (1963, Maps and Diagrams. Methuen, London). A y B son coras en su interacción espacio-temporal. 